\title{
Tempo médio de hospitalização e categorias diagnósticas em hospital psiquiátrico
}

\author{
Mean hospitalization time and diagnostic \\ categories in a psychiatric hospital \\ José Carlos Souza,2, Neomar Souza', Luís Alberto Magna²
}

\section{RESUMO}

Objetivo: Avaliar o tempo médio de hospitalização (TMH) e os diagnósticos dos pacientes. Métodos: Fez-se um levantamento documental dos registros de 2.247 prontuários de pacientes internados de janeiro a dezembro de 2004 em um hospital psiquiátrico. Foram investigados TMH, idade, sexo, procedência e grupos diagnósticos. Usaram-se medidas de tendência central e de dispersão, testes t de Student, de Kruskal-Wallis ou análise da variância, qui-quadrado e de Pearson. Adotou-se o nível de significância de 5\%. Resultados: Eram procedentes de Campo Grande (MS) 59,8\% dos pacientes, 65,5\% eram do sexo masculino ( $p=0,000)$ e 43,3\% apresentavam diagnóstico de esquizofrenia; não houve diferença significativa na idade entre os dois sexos ( $p=0,080)$. O TMH foi de 27,66 dias por paciente. Houve diferença significativa do TMH em função das características diagnósticas $(p=0,001)$ entre os três grupos de procedência ( $p=0,045$ ), ficando as cidades a mais de $200 \mathrm{~km}$ de Campo Grande com média maior de dias de hospitalização (29,1 dias). Também foram encontradas diferenças estatísticas na distribuição das categorias diagnósticas em função do número de hospitalizações $(p=0,000)$, na distribuição das principais categorias diagnósticas $(p=0,000)$ e também nas distribuições em relação à procedência $(p=0,000)$. Conclusão: $\mathrm{O}$ TMH está dentro da média recomendada pelo Ministério da Saúde brasileiro.

\section{ABSTRACT}

Objective: The objective of this study was to evaluate mean hospitalization time (MHT) and patient diagnosis. Methods: Records of 2,247 patients admitted to a psychiatric hospital from January to December 2004 were reviewed to retrieve MHT, age, sex, origin and diagnostic categories. Measures of central tendency and dispersion, Student's t, Kruskal-Wallis or analysis of variance, chi-square and Pearson tests were used for statistical analyses at a $5 \%$ level of significance. Results: Of all patients, 59.8\% were from Campo Grande (MS), 65.5\% were male ( $p=0.000)$, and $43.3 \%$ had a diagnosis of schizophrenia. No significant differences in age were found between sexes $(p=0.080)$. Overall MHT was 27.66 days. A significant difference in MHT was found in diagnostic categories ( $p=0.001$ ) between the groups of patients from different origins ( $p=0.045)$ : patients from cities farther than $200 \mathrm{~km}$ from Campo Grande had a longer hospital stay (29.1 days). Statistical differences were also found for the distribution of diagnostic categories when analyzed according to number of admissions ( $p=0.000)$, for the distribution of the main diagnoses $(p=0.000)$; and for MHT according to patient origin $(p=0.000)$. Conclusions: The THM is within the acceptable level recommended by the Brazilian Department of Health.

Mental health, length of stay, diagnosis. 


\section{INTRODUÇÃO}

Diante das crescentes transformações do atendimento psiquiátrico em regime hospitalar, há de se atualizar as pesquisas sobre o perfil sociodemográfico, o tempo médio de hospitalização $(\mathrm{TMH})$ e os diagnósticos mais freqüentes dos pacientes, a fim de auxiliar no aprimoramento das novas políticas assistenciais. O que chama a atenção quanto ao movimento de reorientação do modelo assistencial brasileiro no campo da saúde mental é que existe muito discurso, mas as ações para a implementação dos novos serviços apresentam-se de forma muito lenta. Em quase todos os discursos, está bem clara a necessidade da criação de novos modelos assistenciais e a extinção dos hospitais psiquiátricos, mas poucas pesquisas são realizadas para se chegar ao conhecimento do perfil dos pacientes internados em hospitais psiquiátricos, de fundamental importância para a implantação de mudanças nessa nova reorganização da política assistencial.

Tanto a conceituação quanto os cuidados dedicados aos indivíduos com transtornos de comportamento variaram no decorrer dos anos e sempre receberam fortes influências de crenças, costumes, rituais, religião, política e até mesmo dos direitos legais de cada época!.

Para que aconteça investimento na elaboração de políticas públicas de saúde, planejamento de programas de promoção e reabilitação social para os doentes mentais, é preciso que haja estudos epidemiológicos na área. Estes ganharam espaço quando foi percebida a sua significância para a detecção, o controle e a prevenção das doenças que atingem a população. No Brasil, observa-se que o aumento do interesse está relacionado à consolidação do Sistema Único de Saúde (SUS), que, por meio da Lei Orgânica de Saúde no 8.080/1990, aponta a necessidade do emprego da epidemiologia para a efetivação de estratégias, alocação de recursos e implantação de programas².

Segundo Desviat a Organização Mundial da Saúde $(\mathrm{OMS})^{4}$ aponta aumento considerável das doenças mentais, tanto nos países de pouca renda quanto na Europa Ocidental e na América do Norte, em conseqüência do envelhecimento da população, do tipo de vida e do número cada vez maior de pessoas que se expõem às mais diversas situações psicossociais. O prolongamento do desemprego, levar uma vida isolada proveniente da desintegração dos laços sociais e familiares e situações de exclusão social favorecem o aumento da vulnerabilidade à doença mental.

Perante a inexistência de estudos epidemiológicos sobre o TMH em hospitais psiquiátricos do estado de Mato Grosso do Sul (MS), os autores do presente estudo vêm pesquisando e publicando estudos a respeito.

\section{MÉTODOS}

A pesquisa foi realizada no Hospital Psiquiátrico Nosso Lar, de Campo Grande (MS), que dispõe de 160 leitos conveniados ao SUS, do Ministério da Saúde. É um hospital de referência na região e recebe pacientes de todo o estado.

Foi realizado um estudo epidemiológico retrospectivo e de corte transversal mediante o levantamento de dados sobre idade, sexo, naturalidade, procedência, TMH e clínicos (grupos diagnósticos) por meio de análise dos prontuários dos pacientes internados no período de janeiro a dezembro de 2004, totalizando 2.247 hospitalizações.

A escolha por grupos de cidades conforme as distâncias ocorreu de forma arbitrária, seguindo a lógica de considerar o hospital, locus da pesquisa, como pólo de atração para o encaminhamento dos pacientes. Decidiu-se por estabelecer três grupamentos entre os 78 municípios que compõem o estado de Mato Grosso do Sul: o primeiro, centralizado na cidade de Campo Grande e cidades limítrofes, o segundo envolvendo as cidades com distância entre 100 e 200 km de Campo Grande e, em terceiro, as cidades situadas a mais de 200 km de Campo Grande. Foi obtida aprovação do Comitê de Ética em Pesquisa da Universidade Federal de Mato Grosso do Sul.

A estatística foi descritiva e com medidas de tendência central e de dispersão, testes t de Student, de Kruskal-Wallis ou análise da variância (ANOVA), qui-quadrado e Pearson. Adotou-se o nível de significância de $5 \%$ em teste bicaudal $(p<0,05)$.

\section{RESULTADOS}

Eram do sexo masculino 65,5\% dos pacientes $(p=0,000$ ). A idade média foi de 39,1 anos no primeiro diagnóstico. O maior número de internações refere-se à cidade de Campo Grande e cidades limítrofes (63,3\%), seguido das cidades localizadas a mais de 200 km de Campo Grande (30,5\%). Não houve diferença na distribuição do sexo em função da procedência $(p=0,065)$ nem da idade entre os dois sexos: $39,91 \%$ entre as mulheres e $38,64 \%$ entre os homens ( $p=0,080$ ). Também não houve diferença entre a idade e a procedência $(p=0,268)$. A maioria dos pacientes sofreu, no período, quatro internações (95\%), metade sofreu somente uma internação e $75 \%$ até duas internações.

Com relação ao total de categorias diagnosticadas observadas no período referente às 2.247 hospitalizações, que 43,3\% dos diagnósticos são de esquizofrenia (F20); 24,6\% são de transtornos mentais e de comportamento decorrentes do uso de álcool (F10); 7,4\% correspondem a transtorno afetivo bipolar (F31); 5,9\% são de transtornos mentais e de comportamento decorrentes do uso de múltiplas 
Tabela 1. Distribuição do tempo de internação em função das principais categorias diagnósticas.

\begin{tabular}{|c|c|c|c|c|c|c|}
\hline Categoria diagnóstica & $n$ & Média & DP & EPM & Mínimo & Máximo \\
\hline F6 - Outros transtornos mentais decorrentes de lesão e disfunções cerebrais e de doença física & 52 & 27,77 & 9,86 & 1,37 & 7 & 50 \\
\hline F10 - Transtornos mentais e de comportamento decorrentes do uso de álcool & 349 & 27,11 & 9,63 & 0,52 & 3 & 70 \\
\hline F19 - Transtornos mentais e de comportamento decorrentes do uso de múltiplas drogas e uso de outras substâncias psicoativas & 83 & 23,18 & 10,55 & 1,16 & 2 & 49 \\
\hline F20 - Esquizofrenia & 428 & 29,64 & 12,85 & 0,62 & 3 & 87 \\
\hline F31 - Transtorno afetivo bipolar & 90 & 27,16 & 9,91 & 1,04 & 3 & 51 \\
\hline Outros & 180 & 26,99 & 14,66 & 1,09 & 1 & 106 \\
\hline
\end{tabular}

$D P=$ desvio-padrão; $E P M=$ erro-padrão da média; $p=0,001$

drogas e uso de outras substâncias psicoativas (F19); 4,5\% são de outros transtornos mentais decorrentes de lesão e disfunções cerebrais e de doença física (F6); 3,1\% correspondem a transtornos esquizoafetivos (F25); 2,3\% são de episódio depressivo (F30); 1,7\% são de retardo mental moderado (F71); 1,4\% são de transtornos psicóticos agudos e transitórios (F23); e 1,2\% são de episódio depressivo (F32), perfazendo a porcentagem acumulada de $95,6 \%$ das categorias diagnosticadas.

O sexo não influenciou significativamente o tempo de internação, ficando a média entre as 408 mulheres em 28,17 dias (desvio-padrão [DP] = 12,28 dias), e em 27,55 dias $(\mathrm{DP}=11,72$ dias) entre os 774 homens $(p=0,390)$. O tempo de internação também não foi influenciado pela idade. A tabela 1 mostra os dados referentes ao tempo de internação em função das principais categorias diagnósticas.

A tabela 2 apresenta os valores médios do tempo de internação, em dias, de acordo com a procedência.

Há diferença significativa do tempo de internação entre os três grupos $(p=0,045)$, decorrente do maior tempo de internação entre os pacientes procedentes de cidades localizadas a mais de 200 km de Campo Grande em comparação àqueles de Campo Grande e cidades limítrofes $(p<0,050)$. O número de internações, em média, foi de $1,93(\mathrm{DP}=1,38)$ entre as 408 mulheres e 1,89 ( $D P=1,28$ ) entre os 774 homens, sendo considerados semelhantes ( $p=0,630)$. A idade não influenciou o número de internações, já que não houve correlação significativa entre ambas $(r=0,04 ; p=0,160)$. A procedência não influenciou o número de internações $(p=0,927)$.

Houve significativa diferença na distribuição das categorias diagnósticas em relação à internação múltipla (duas ou mais) ou única. Esta diferença deve-se à maior freqüência de ocorrências de transtornos mentais e de comportamento decorrentes do uso de álcool (F10) e outros diagnósticos não listados na internação única em relação a múltiplas, dando-se o contrário em relação aos diagnósticos de esquizofrenia (F20) e transtorno afetivo bipolar (F31).

\section{DISCUSSÃO}

Quanto à procedência dos 2.247 pacientes, de acordo com a cidade de origem, 59,8\% dos pacientes são de Campo Grande (MS). Brenner et al. ${ }^{5}$, em estudo realizado na cidade de Porto Alegre (RS), em um hospital psiquiátrico, relataram que $54,2 \%$ dos pacientes eram procedentes de Porto Alegre, 18,2\% residiam no interior do estado do Rio Grande do Sul e $27,5 \%$ eram oriundos de outros estados. Correia ${ }^{6}$ demonstrou, em estudo sobre internação psiquiátrica na região de Ribeirão Preto (SP) realizado em quatro hospitais, que a grande maioria de pacientes internados residia na cidade de Ribeirão Preto ou nas cidades vizinhas.

A maioria dos pacientes deste estudo sofreu até quatro internações, sendo que a metade sofreu somente uma internação e 75\% duas internações. Observou ainda que, nos quatro hospitais da região de Ribeirão Preto, existem taxas superiores a $67 \%$, um deles apresentava taxa de $22,2 \%$ de pacientes com somente uma internação. Quanto às reinternações nos referidos hospitais, foram de 33\% no hospital Z; $31,4 \%$ no hospital W; $23,1 \%$ no hospital Y; e 77,8\% no hospital X. Bassit (1981 apud Correia ${ }^{6}$ ), em uma pesquisa em hospital psiquiátrico em Tremembé (SP), encontrou taxa de $72 \%$ referente à reinternação dos pacientes, e 28\% dos casos eram de primeiras internações, diferindo do atual estudo.

A esquizofrenia é o diagnóstico de maior prevalência no presente estudo $(43,3 \%)$, corroborando com outros realizados em hospitais psiquiátricos, tanto nacionais quanto internacionais. Coutinho et al.7, em um estudo mais amplo

Tabela 2. Valores médios do tempo de internação, em dias, de acordo com a procedência.

\begin{tabular}{|c|c|c|c|c|c|c|}
\hline Categoria diagnóstica & n & Média & DP & EPM & Mínimo & Máximo \\
\hline Campo Grande e cidades limítrofes & 745 & 27,15 & 11,41 & 0,42 & 1 & 81 \\
\hline Cidades distantes de $100 \mathrm{~km}$ a $200 \mathrm{~km}$ de Campo Grande & 76 & 27,66 & 9,78 & 1,12 & 10 & 62 \\
\hline Cidades a mais de $200 \mathrm{~km}$ de Campo Grande & 361 & 29,06 & 13,2 & 0,69 & 3 & 106 \\
\hline
\end{tabular}

$D P=$ desvio-padrão; $E P M=$ erro-padrão da média; $p=0,045$. 
em 20 hospitais psiquiátricos do Rio de Janeiro (RJ), envolvendo a participação de 3.223 pacientes internados, encontrou como diagnóstico mais freqüente a esquizofrenia (42,1\%). Já em outro estudo, realizado também por Coutinho et al. ${ }^{7}$ em uma instituição asilar no Rio de Janeiro incluindo 1.494 participantes, os diagnósticos mais freqüentes foram a esquizofrenia (53,6\%), o retardo mental (26,4\%) e o alcoolismo (1,8\%), diferindo dos anteriores e, inclusive, do presente estudo. Dhadphale e Shaikh ${ }^{8}$, em estudo realizado no Zâmbia, analisaram o diagnóstico psiquiátrico em uma amostra de pacientes hospitalizados e constataram que a maioria (27\%) era portadora de esquizofrenia. Em um estudo realizado em Madri (Espanha), no Hospital Punta de Europa, de Algeciras, cujo objetivo foi descrever a freqüência de reingressos, foi constatado que, das reinternações psiquiátricas, a esquizofrenia era a responsável pela maior parte das readmissões, o que se verificou também no atual estudo. Já no estudo de Ballón et al. ${ }^{9}$, realizado no Hospital Nacional Edgardo Rebagliati Martins de La Seguridad Social del Perú (HNERM-ESSALUD), 40\% dos pacientes atendidos nos diversos serviços do departamento de saúde mental do HNERM-ESSALUD, no ano de 2000, tiveram diagnóstico de esquizofrenia. Esses resultados estão muito próximos dos encontrados neste estudo. Nos estudos de Brenner et al. ${ }^{5}$, no Rio Grande do Sul, Caton e Gralnick ${ }^{10}$, nos Estados Unidos, Coutinho et al. ${ }^{7}$, no Rio de Janeiro, e Oksenberg et al. ${ }^{11}$, no Chile, o diagnóstico de esquizofrenia foi responsável por 40\% a 60\% do total das internações. Para Brenner et al. ${ }^{5}$, o diagnóstico de esquizofrenia foi relatado para 67,1\% dos pacientes. Menezes e Mann ${ }^{12}$, em um estudo sobre o ajustamento social de pacientes com esquizofrenia, relatam que a esquizofrenia é um grave problema de saúde pública em países desenvolvidos, afetando, em média, 0,5/1.000 pessoas adultas e provocando altos custos econômicos para a sociedade. Relatam, também, que, no Brasil, o diagnóstico de esquizofrenia representa cerca de um terço de todas as internações psiquiátricas e que existe carência de informações sobre as reais condições e perfil desses pacientes.

No presente estudo, o diagnóstico de transtornos mentais e de comportamento decorrentes do uso de álcool (F10) ocupa o segundo lugar (24,6\%), mostrando similaridade com outros estudos. No estudo de Hambrecht e Häfner ${ }^{13}$, o abuso de substâncias é considerado problema comum em pacientes esquizofrênicos, ocorrendo em até 60\%. Outro estudo realizado no Hospital Central Militar, na Cidade do México, por Ramos-Pinedo e Hernández-Daza ${ }^{14}$ para verificar o consumo de álcool em pacientes psiquiátricos (comorbidade), utilizou-se uma amostra de 105 pacientes internados em período de seis meses (setembro de 1998 a fevereiro de 1999). A associação encontrada entre os transtornos psiquiátricos e o consumo de álcool foi de 27,3\% com os transtornos esquizofrênicos.
No presente estudo, o TMH foi semelhante entre homens e mulheres, ficando em 27,55 dias entre os 774 pacientes do sexo masculino e em 28,17 dias entre as 408 mulheres. Porém, há na literatura discrepâncias muito significativas no TMH em hospital psiquiátrico. Há de se considerar que as metodologias dos censos são bem diversificadas, assim como a legislação e o controle sanitário local. Também é preciso considerar o tipo e as características peculiares de cada hospital. O TMH estabelecido pelo Ministério da Saúde brasileiro é de 30 dias para uma internação de adulto $^{15}$. Brenner et al. ${ }^{5}$ detectaram um tempo de internação de 30 dias em uma amostra de 258 homens e 225 muIheres em um hospital de Porto Alegre (RS). Lieberman et al. ${ }^{16}$ estudaram 217 pacientes e encontraram um TMH de $23 \pm 16,2$ dias. Hibbard e Trimboli17 encontraram média de 21 dias em 612 pacientes. Caton e Gralnick ${ }^{10}$ estudaram 119 pessoas com esquizofrenia crônica de três hospitais e encontraram a variação de 21 a 70 dias. No estudo de Mattes et al. ${ }^{18,}$ chegou-se ao extremo de 179 dias. Coutinho et al. ${ }^{7}$ estudaram 1.494 pacientes internados em um hospital psiquiátrico do Rio de Janeiro, com o predomínio de homens $(53,4 \%)$, e encontraram $77,6 \%$ com o tempo de internação $\geq$ 2 anos. Os mesmos autores, em um estudo mais amplo em 20 hospitais psiquiátricos do Rio de Janeiro, inclusive os de longa duração, totalizando 3.223 pacientes (66\% homens), relataram que mais da metade estava internada há mais de três meses, e um terço há mais de um ano.

Neste estudo houve predomínio do sexo masculino nas internações. Coutinho et al. ${ }^{7}$ também relataram, em seu estudo, o predomínio do sexo masculino $(53,4 \%)$ sobre o sexo feminino (46,6\%). Herrera ${ }^{19}$ concluiu, em seu trabalho com pacientes com esquizofrenia internados no hospital psiquiátrico Julio Endara (Quito, Equador), em 1995, que a maioria dos pacientes internados era do sexo masculino (63,3 versus $36,6 \%$ do sexo feminino).

A faixa etária predominante em estudos similares é variável. Amarante et al. ${ }^{20}$, em um censo dos pacientes internados em 20 hospitais psiquiátricos do Rio de Janeiro, encontraram faixa etária inferior a 40 anos, corroborando este estudo. Brenner et al. ${ }^{5}$ observaram, em seus estudos com uma amostra de 258 homens e 224 mulheres, que 28,4\% de ambos os sexos tinham idade entre 31 e 40 anos. Correia ${ }^{6}$, em seu estudo, relata que grande concentração de pacientes se situa na faixa etária entre 20 e 49 anos, diferindo dos dados deste estudo.

\section{CONCLUSÃO}

O TMH está dentro da média recomendada pelo Ministério da Saúde brasileiro, porém urgem novos estudos para contribuir na elaboração de políticas e ações de saúde mental cada vez mais eficazes e menos burocratizadas. 


\section{REFERÊNCIAS}

1. Resende H. Política de saúde mental no Brasil: uma visão histórica. In: Tundis AS, Costa NR (orgs.) Cidadania e loucura: políticas de saúde mental no Brasil. 7. ed. Petrópolis: Vozes, 2001. p.15-69.

2. Organização Pan-Americana de Saúde. Organização Mundial da Saúde. Relatório sobre saúde no mundo 2001: saúde mental - nova concepção, nova esperança. Genebra, 2001.

3. Desviat M. A reforma psiquiátrica. Rio de Janeiro: Fiocruz, 2002.

4. Organização Mundial da Saúde. Relatório da Organização Mundial da Saúde: doenças mentais atingem 450 milhões de pessoas. Brasília, 2001.

5. Brenner MK, et al. Mudanças na clientela de internados em hospital psiquiátrico. J Bras Psiquiatr. 1999;48(9):415-20.

6. Correia VR. Internação psiquiátrica na região de Ribeirão Preto no período de 1989 a 1993. 1998. 96 f. [Dissertação]. Escola de Enfermagem de Ribeirão Preto, Universidade de São Paulo. Ribeirão Preto, 1998.

7. Coutinho ESF, et al. Censo de pacientes internados em uma instituição asilar no estado do Rio de Janeiro: dados preliminares. Cad Saúde Pública. 2002;18(6):1803-7.

8. Dhadphale M, Shaikh SP. Psychiatric diagnosis of 236 admissions seen at Ndola, Zambia. East African Medical Journal. 1981;58(1):65-71.

9. Ballón R, et al. Causas de rehospitalizacion de pacientes esquizofrênicos crónicos. Rev Neuro-psiquiatr. 2003;66(1):13-26.

10. Caton CLM, Gralnick A. A review of issues surrounding length of psychiatric hospitalization. Hosp Community Psychiatry. 1987;38(8):858-63.
11. Oksenberg A, et al. Descripción de variables que inciden sobre el tiempo de hospitalización en el Hospital Psiquiátrico José Horwitz Barak. Rev Psiquiatr. (Santiago de Chile). 1989;6(2):127-36.

12. Menezes PR, Mann AH. The social adjustment of patients with schizophrenia: implications to the mental health policy in Brazil. Rev Saúde Pública. 1993;27(5):340-9.

13. Hambrecht $M$, Häfner $H$. Substance abuse and the anset of schizophrenia. Biological Psychiatry. 1996;40:1155-63.

14. Ramos-Pinedo A, Hernández-Daza M. Consumo de alcohol en los pacientes psiquiátricos del Hospital Central Militar: determinación de factores predictivos de riesgo. Revista de Sanidad Militar. 2000;54(4):200-3.

15. Brasil, Ministério da Saúde. Orientações para funcionamento e supervisão dos serviços de saúde mental. Brasilia, DF, 1990.

16. Lieberman PB, et al. Dimensions and predictors of change during brief psychiatric hospitalization. General Hospital Psychiatry. 1993;15(5):316-24.

17. Hibbard T, Trimboli F. Correlates of successful short-term psychiatric hospitalization. Hosp Community Psychiatry. 1982;33:829-33.

18. Mattes JA, Rosen B, Klein DF. Comparison of the clinical effectiveness of "short" versus "long" stay psychiatric hospitalization, Il: results of a three-year post-hospital follow-up. The Journal of Nervous and Mental Disease. 1977;165(6):387-94.

19. Herrera F. Incidência de esquizofrenia em el hospital psiquiátrico Julio Endara, 1995. Rev Fac Cien Méd (Quito). 1997;22(1):36-9.

20. Amarante PD, Coutinho ESF, Silva JPL. Perfil demográfico e sócio-econômico da população de internos dos hospitais psiquiátricos da cidade do Rio de Janeiro. Cad Saúde Pública. 1999;15(3):505-11. 